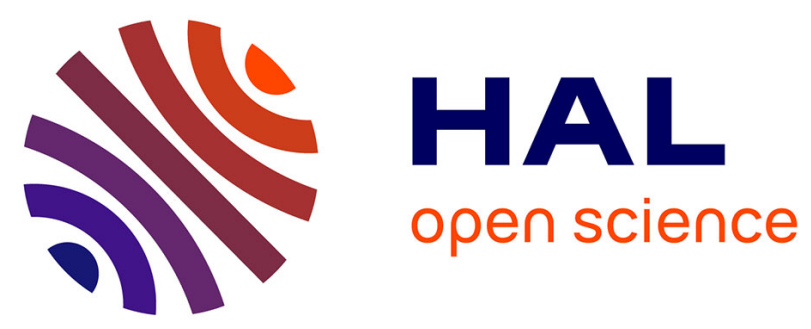

\title{
Les gares au miroir de l'urbain
}

\author{
Nacima Baron-Yelles, Nathalie Roseau
}

\section{To cite this version:}

Nacima Baron-Yelles, Nathalie Roseau. Les gares au miroir de l'urbain. Flux - Cahiers scientifiques internationaux Réseaux et territoires, 2016, Les gares au miroir de l'urbain, 103-104, pp.1-8. 10.3917/flux.103.0001 . hal-01343076

\section{HAL Id: hal-01343076 https://hal.science/hal-01343076}

Submitted on 7 Jul 2016

HAL is a multi-disciplinary open access archive for the deposit and dissemination of scientific research documents, whether they are published or not. The documents may come from teaching and research institutions in France or abroad, or from public or private research centers.
L'archive ouverte pluridisciplinaire HAL, est destinée au dépôt et à la diffusion de documents scientifiques de niveau recherche, publiés ou non, émanant des établissements d'enseignement et de recherche français ou étrangers, des laboratoires publics ou privés. 


\title{
Les gares au miroir de l'urbain
}

\author{
Nacima Baron \\ Nathalie Roseau
}

\begin{abstract}
« Partir par la gare, c'est, en effet, constituer d'une façon privilégiée, la ville comme une totalité. [...] Dans la gare, avant de partir, nous avons le sentiment de tourner le dos à toute la ville, encore présente et non point réduite à une représentation lointaine. En effet, la gare comme nous le verrons par la suite, est en dehors et en dedans de la ville. Nous pouvons alterner les points de vue : assez proches d'elle pour être assurés de son existence, assez distants pour la globaliser du dehors. »
\end{abstract}

Pierre Sansot, 1971, Poétique de la Ville, Paris : Klincksieck, p. 85

L e choix d'offrir au lecteur un nouvel ensemble de recherches sur les gares se doit d'être explicité. Leur omniprésence dans les discours sur l'aménagement est précisément à l'origine du séminaire doctoral dont est issu ce numéro de Flux. Suscitant un sentiment de saturation, ces discours placent les gares - celles qui se réaménagent ou s'inventent en lien avec le renouveau de la grande vitesse, avec le programme du Grand Paris, avec la croissance des mobilités - en articulation étroite avec les interrogations associées aux dynamiques urbaines et métropolitaines: espace public, pratiques, densification, suburbanité, gouvernance. Problème et promesse en même temps, lieu de mobilisation, la gare constitue un point focal des refondations urbaines comme des contestations sociales. Toutefois, l'effervescence observée dans la production d'ateliers, de débats, de manifestations, de publications, crée aussi un métadiscours qui peut faciliter des approches déterministes des liens entre la ville et la mobilité, en dépit de l'avertissement lancé il y a plus de vingt ans par Offner (1993) sur le mythe des effets structurants des infrastructures de transport. Tous réflexes dont nous souhaiterions ici nous départir en interrogeant les relations qui unissent la gare et l'urbain selon un angle double, d'abord de leur opportunité, en lien avec l'actualité de l'aménagement et de l'urbanisme, ensuite de l'orientation épistémologique que nous proposons.

\section{LA GARE, MATRICE URBAINE}

La relation que nouent la gare et l'urbain conduit tout d'abord à réinterroger cet artefact technique et territorial dans sa dynamique temporelle longue. La gare marque fortement l'émergence dans la deuxième moitié du XIX ${ }^{e}$ siècle, d'une métropole des techniques, d'une industrie des réseaux, d'une société de l'événement et de l'avènement (Chéroux, 1996 ; Bowie, Texier, 2003 ; Desportes, 2005 ; Sauget, 2009). Les sens y sont frappés par l'impression d'accélération. « La mer du Nord déferle au seuil de ma porte » écrit le parisien d'adoption Heinrich Heine peu après la mise en service des premières grandes lignes européennes. Et le baron Joseph Von Eichendorff d'ajouter que " ces voyages à la vapeur mêlent continuellement les uns à travers les autres, à la manière d'un kaléidoscope, les objets d'un monde qui, pourtant, à la vérité, ne se compose plus que de gares [...] » (Schivelbusch, 1990, p. 43).

Le temps long de l'histoire invite à considérer la plasticité de la gare dans l'espace urbain, à la fois dans ses permanences et ses mutations. Surtout, le paradigme moderniste de la gare qui jalonne son histoire mobilise un imaginaire que les promesses actuelles recyclent, nourrissant des souvenirs, les transformant et les réinsérant dans un nouvel ensemble d'images et de valeurs qui se construisent à partir d'exemples et d'échos de 
gares circulant les uns vers les autres. La gare peut ainsi être lue comme un palimpseste des représentations successives qu'y ont projetées les sociétés urbaines, sa complexité procédant tout autant de la matérialité qui la constitue que de l'imaginaire qui la fonde (Roseau, 2012).

Entre résurgence d'un passé et préfiguration des lendemains, les gares tracent leur trajectoire. Les batailles patrimoniales autour des projets de réinvestissement de grandes gares historiques comme celles de Stuttgart, Saint Pancras à Londres ou Grand Central à New York - sur laquelle un article du présent dossier reviendra - illustrent bien les mobilisations et les conflits de références sur le devenir de lieux à forte valeur symbolique (1). Car si les gares constituent un patrimoine, elles sont aussi un laboratoire in vivo de la production urbaine contemporaine. Ce faisant, elles dévoilent une succession de scènes ou d'arènes dans lesquelles s'affrontent des représentations, des discours, des stratégies relevant de logiques politiques, économiques, sociales véritablement intriquées.

C'est toute cette complexité que ce numéro sur les gares entend interroger, autour d'une problématique qui approfondit la nature des relations qui unissent la ville à la gare, la figure du miroir nous guidant dans cette investigation. Quels sont les effets d'imitation, de déformation, de distanciation, de révélation, de condensation générés par les tensions qui aimantent sur le temps long de l'histoire les deux termes de ce couple ? De quelle façon la gare apparaît-elle comme matrice urbaine des apories et des tentations qui façonnent la ville ? D'une part, la gare semble capter les tensions qui travaillent l'urbanité, au point qu'elles cristallisent dans son microcosme. D'autre part, le champ de l'urbain s'acculture au contact des gares dont l'imaginaire, les modèles et les processus d'aménagement agissent sur sa transformation. Comme le soulignait Pierre Sansot (1971), " la gare est devenue un modèle. On a cru ensuite reconnaître des librairies, des brasseries, des horloges qui ressemblaient à des librairies, à des brasseries, à des horloges de gare, tout comme certaines générations ont eu l'impression de rencontrer dans la vie des personnages de Balzac ou de Musil. » (p. 91).

C'est ce double mouvement d'aspiration et de diffusion que nous souhaitons interroger, dans son fonctionnement et ses effets, au travers des divers articles qui suivent. Ceux-ci sont issus de communications faites lors d'un séminaire doctoral qui s'est engagé en 2013, dans le cadre de la Chaire Gares créée au sein de l'École nationale des ponts et chaussées (2). Son programme de recherche s'inscrivait dans le champ des études urbaines et des sciences sociales - géographie, aménagement, sciences politiques, sociologie, sémiologie mais aussi histoire urbaine. Il entendait en particulier donner la parole à de jeunes chercheurs dont les travaux doctoraux se consacrent aux gares. Les huit séances du séminaire ont ainsi permis de confirmer I'hypothèse d'une forte dynamique de recherche et de dessiner un espace de connaissance sans prétention aucune à l'exhaustivité (3). En multipliant les coups de sonde dans un corpus de travaux en cours qui frôle la cinquantaine de thèses au niveau national, en ouvrant la parole à des horizons problématiques divers - de l'urbanité à l'habitabilité, de la surveillance au langage, de la modernité au patrimoine -, le séminaire a permis d'élaborer de multiples variations sur un ensemble de thèmes assez convergents, qui prolongent et renouvellent une historiographie déjà longue et dense.

\section{LES TEMPS DES GARES, UN ÉTAT DE LA RECHERCHE en France}

Le Temps des gares renvoie au titre d'une exposition tenue au Centre Georges Pompidou en 1978, à l'époque où s'engageait la construction d'un réseau express régional (RER) à travers Paris et dans la banlieue proche. Avec l'ouvrage de l'exposition (Dethier, 1978) est aussi publié un numéro de la revue du Centre de création industrielle Traverses (1978) regroupant des contributions de Paul Virilio, Pierre Sansot, Michel de Certeau, Marc Ferro, Louis Marin, Gérard Monnier. Antérieurement à I'exposition, des recherches avaient été réalisées, marquantes quoique très isolées à l'instar de la thèse soutenue par le géographe René Clozier sur la Gare du Nord en 1940 : « La gare est le monument le plus représentatif des métropoles modernes, le symbole du brusque accroissement démographique et économique, déclenché par la « révolution » des chemins de fer. La gare est donc un phénomène urbain et, comme tel, relève de la recherche géographique » (p. 15). Déjà Clozier s'interrogeait sur la transversalité de l'objet pour la recherche urbaine, ou sur la manière de faire terrain dans une gare.

Pour la scène ferroviaire, la fin des années 1970 marque un tournant essentiel : technologique avec le lancement du TGV et la construction de lignes et de nouvelles gares, fonctionnel avec l'événement symbolique de la suppression du ticket de quai, commercial avec l'extension des activités extra-ferroviaires (restauration, services, commerces), patrimonial avec l'affirmation légitime de la monumentalité des gares. À cette date, l'enjeu des gares se mesure aussi à leur capacité à 
affronter la concurrence automobile. En France, les recettes du trafic des voyageurs grandes lignes progressent. L'automobile semble avoir vécu son âge d'or alors que la crise pétrolière a déjà frappé et que la conscience écologique mûrit. Ce retour en force du ferroviaire au cœur des débats d'actualité sur la politique des transports, mis en perspective avec l'initiative fédérale aux États-Unis de reconstruire avec Amtrak un réseau de lignes de chemin de fer, conduit à mettre la gare sous les feux des projecteurs, à laquelle le commissaire de l'exposition Le Temps des Gares, Jean Dethier (1978) dédie cet hommage : "La gare est un véritable sismographe des vibrations et convulsions de notre société, de son dynamisme ou de son déclin, de ses moindres fluctuations. [...] Resterons-nous indifférents aux paradoxes de cette destinée, où se conjuguent le destin et le progrès, où se résume, telle Babel en son temps, la confusion des élans de notre société ? » (p. 12-13). L'exposition du Centre Pompidou circule, présentée à York et Londres en 1981 sous le titre All Stations (Dethier, 1981), à Bruxelles, Amsterdam, Milan. Son catalogue est traduit en quatre langues, attestant de I'internationalisation de son objet d'études. Cette actualité du renouveau des gares se prolonge en France dans une série de travaux scientifiques publiés par des historiens comme Bowie (1987, 1996), Lemoine (1994) et Ribeill $(1993,1996)$ ainsi que par d'autres chercheurs (Dupuy, 1993 ; Joseph, 1996 ; Sander, 1995).

Au tournant des années 2000 s'ouvre un nouveau moment pour la recherche urbaine sur les gares. Gallimard inaugure cette période avec la publication d'un guide sur La France des Gares (2000). Élevée au rang de patrimoine national, touristique et culturel, immatériel et matériel, la gare fait l'objet d'ouvrages portant sur la typologie architecturale (Duthilleul, Tricaud, 1999 ; Mazzoni, 2001) puis, plus monographiques, sur une gare remarquable en particulier (Bradley, 2011 ; Belle, Leighton, 2012). Dans le sillage des travaux de Ribeill, se publient des travaux de recherche critiques inscrits dans le champ des sciences humaines et sociales. Les revues des Annales de la Recherche Urbaine (1996) et des Annales des Ponts et Chaussées (1999) publient chacune un numéro spécial consacré aux gares et à leur urbanisation. À la suite de ses travaux engagés au début des années quatre-vingt dix sur la Gare du Nord avec la RATP (Régie autonome des transports parisiens) et la SNCF (Société nationale des chemins de fer), Isaac Joseph explore ce que Georges Amar (alors responsable de l'unité prospective de la RATP) nomme les «lieux-mouvements » de la ville, en scrutant les espaces et les pratiques corporelles et discursives des voyageurs (Joseph, 1995, 1999). Sauget (2009) aborde ensuite la gare selon une histoire sociale et culturelle des représentations. La recherche s'internationalise enfin, donnant lieu à des études approfondies sur les gares japonaises par exemple (Aveline-Dubach, 2003 ; Doumas, 2008), ou de nouveaux concepts d'aménagement comme les Transit Oriented Development (TOD) (Pucci, 1999 ; Grillet-Aubert, 2015).

De manière concomitante, les institutions s'emparent du sujet. Les programmes de recherche ministériels investissent les " gares et quartiers de gares » à l'instar du PUCA (Plan urbanisme, construction, architecture) qui initie une plateforme d'observation des projets et stratégies urbaines, dont les gares font partie (Terrin, 2011). Se développent également des travaux de nature projectuelle ou appliquée, à l'initiative de I'Institut d'aménagement et d'urbanisme de la Région îlede-France, l'Atelier Parisien d'Urbanisme, la Société du Grand Paris, I'Atelier International du Grand Paris, la SNCF, la RATP et d'agences d'architecture et d'urbanisme associées aux démarches du Grand Paris. Les publications grand public, mais aussi les séminaires et groupes de travail se multiplient, au moment où la gare, après un relatif effacement, revient à l'agenda des politiques publiques et de la planification urbaine.

Cette floraison signale le tournant dont les gares sont l'objet, marquant à la fois l'aboutissement d'un cycle - celui de la grande vitesse - et l'amorce de sa maturité dans un contexte de massification des trafics régionaux et suburbains - le mass transit. Si la grande vitesse connaît un deuxième souffle avec les projets des lignes Rhin-Rhône, Sud Atlantique et des connexions transfrontalières avec les réseaux espagnol, suisse et italien, la gare est davantage revisitée dans le cadre de la modernisation des réseaux ferroviaires urbains et régionaux. La convergence et la massification des mobilités intra et interurbaines conduisent à repenser les gares régionales - Bordeaux Saint-Jean, Montpellier Saint-Roch, Toulouse Matabiau... Le lancement du programme du Grand Paris Express et les projets de rénovation de lignes régionales participent d'un ample mouvement de réinvestissement des gares urbaines et suburbaines (4). Ce nouvel avènement des gares est lui-même étroitement lié à une série de facteurs structurants qui remodèlent l'environnement des gares. L'ouverture à la concurrence dans le domaine ferroviaire a enclenché un processus de libéralisation qui perfuse les organisations et leur fonctionnement, et produit plus largement un mouvement de 
commercialisation/valorisation de l'espace ferroviaire (Riot, 2015). Le processus de métropolisation - intensifié par les logiques de valorisation foncière et immobilière, les restructurations dans le champ des services, du commerce et des nouvelles technologies - induit par ailleurs une recapitalisation et une revalorisation multiforme de l'environnement des gares.

\section{ESPACE, FONCTION, REPRÉSENTATION : LA GARE NEXUS URBAIN}

Le titre "Les gares au miroir de l'urbain » peut se comprendre dans un premier temps comme l'expression d'une hypothèse simple : lieu du transport, la gare est aussi attracteur et condensé d'urbanité. Cette acception du rôle de la gare dans le cadre urbain doit être à nos yeux interrogée sans dissocier trois dimensions qui s'enchevêtrent et composent l'horizon de la gare.

La première dimension est d'ordre spatial : la gare constitue un assemblage complexe d'espaces et de volumes imbriqués, couverts et bâtis ou non, plus ou moins récents ou renvoyant au contraire à une empreinte ancienne - fuseaux, aiguillages. Ce système technique est associé à des régimes de propriété foncière, à des formes d'exploitation, à des registres d'usages dans lesquels la dimension publique et ouverte est somme toute restreinte : le bâtiment voyageur, le parvis, les espaces de stationnement. Au-delà de son enceinte proprement dite, il faut interroger l'empreinte que la gare détermine dans son quartier. La gare de Shinjuku à Tokyo, plus grande gare du monde avec près de 4 millions de visiteurs chaque jour, constitue à elle seule un ensemble d'ouvrages et d'immeubles, une mégastructure, une mosaïque de quartiers, une ville intérieure et souterraine. La définition de ce qui fait l'intériorité et l'extériorité de la gare, de ce qui délimite ses frontières et ses seuils est tout sauf évidente. La multiplicité des enveloppes de la gare et la complexité de l'organisation des espaces associés permettent d'interroger selon différents angles les liens entre l'espace de la gare et l'espace de la ville.

La deuxième dimension est d'ordre fonctionnel : la gare accueille des usages qui n'ont pas spécifiquement à voir avec la finalité ferroviaire, mais procèdent de son rôle d'attracteur de flux plus ou moins captifs. L'intensification des activités et des mobilités dans les quartiers de gares s'appuie sur une offre intermodale dense, qui en retour déclenche une attractivité de la gare: des foules de plus en plus nombreuses transitent dans les gares, déclenchant l'intérêt d'opérateurs de plus en plus diversifiés - publicitaires, acteurs du commerce de détail, professionnels de l'immobilier, des filières agro-alimentaires et de la restauration - pour capter et « fertiliser » ces flux piétonniers. S'appuyant sur des coalitions d'acteurs publics et privés, les gares et leurs quartiers participent enfin d'un cycle de revalorisation foncière, contribuant aux processus urbains et métropolitains.

La troisième dimension renvoie enfin à la question des représentations: la gare peut être lue comme un microcosme d'images et de perceptions fortement articulé avec le macrocosme urbain (Wucherpfennig, 2006). Dès son invention au XIX ${ }^{\text {e }}$ siècle, elle représente une sorte de "condensat » de la modernité citadine. Dans l'espace contemporain de la gare, le règne de la mobilité comme valeur dominante a favorisé I'omniprésence de systèmes de signes qui façonnent l'espace public : déploiement d'une signalétique du mouvement ; prolifération de symboles valorisant la vitesse, l'ubiquité, l'immédiateté, la proximité ; généralisation d'objets technologiques. En même temps, la gare est un lieu d'ancrage pour des populations ou des activités qui lui confèrent un rôle de place urbaine. Espace de flux massifs et routiniers, elle est un lieu de séjour, cinétique ou statique, qui génère des pratiques locales et spatiales. Enfin, la gare produit des urbanités. Elle dispense des atmosphères métropolitaines, à travers l'expérience de la foule ou les environnements sensoriels. Elle est un lieu de frottement entre les individus de la métropole.

\section{UN KALÉIDOSCOPE}

La figure du miroir renvoie d'abord au dispositif optique qui permet de scruter et de mettre à distance le sujet observé. Dès I'avènement du chemin de fer, le choc mécanique et sensitif de I'entrée du train en ville conduit peintres et écrivains à adapter leur lentille d'observation. Intégrant le rail dans leurs œuvres de fiction, Claude Monet ou Émile Zola se documentent précisément, scrutent le réel qui survient, puis créent un réel de fiction qu'ils exacerbent dans des œuvres et des romans à forte dimension sociale (Van Weddingen, 2004) (5). Pour figurer la Gare Saint-Lazare (1877), Claude Monet effectue des recherches précises sur ses modèles ferroviaires. En même temps, sa vue saisissante renvoie à un univers fantastique dont les brumes et les fumées, les coexistences de matières et d'échelles rendent compte de manière spectaculaire, fixant le face-à-face de la métropole et du chemin de fer, de la foule et de la machine. S'installant lui aussi à Saint-Lazare, Émile Zola 
utilise un procédé similaire pour l'écriture de La Bête Humaine allant jusqu'à décrire un modèle de société imbriqué dans la société traditionnelle telle qu'elle existe en 1890. Le réalisme de sa fresque procède des dix années qu'il mit pour préparer son roman, documentant, éprouvant par des descriptions, des témoignages, ce rapport au réel. Dans I'adaptation cinématographique qu'il fait en 1938 du roman, Jean Renoir se veut fidèle au naturalisme de l'écrivain plus qu'à sa narration. Fruit d'un long travail de recherche préparatoire, le cinéaste dresse une fresque de la vie des cheminots avec une exactitude quasi documentaire. Déployant à partir de la géographie ferroviaire, un espace imaginaire, lieu de l'intrigue dramatique, il dresse un tableau social de son époque, inscrivant l'œuvre de Zola dans le présent (6).

Plus récemment, la double production du film et du documentaire de Claire Simon, Gare du Nord, témoigne d'une enquête préparatoire très fouillée, allant de l'observation fine des lieux au casting des « acteurs » de la gare, montrant le film comme un document où se noue une intrigue, et le documentaire comme un film qu'habitent les usagers de la gare (Simon, 2013 a, b). Cette frontière ténue, le cinéaste Terry Gillian l'avait déjà exprimée dans son film The Fischer King (1991), qui raconte l'histoire d'un arriviste et d'un clochard tous deux à la recherche du Graal, et fait jouer plus de mille figurants dans une scène mémorable tournée à Grand Central Station : «Peu avant 5 heures de l'après-midi, relate-t-il, le rythme de Grand Central devient plus intense. C'est génial, il y a de plus en plus de gens, ils marchent de plus en plus vite, je me suis dit que ça serait merveilleux de les faire valser, tous ensemble, le temps d'un instant, avant de les voir repartir vers leurs trains » (7). Dans cette chorégraphie ferroviaire, la gare figure un lieu où fiction et réel coexistent, s'entremêlent et s'inversent.

Mais la gare n'est pas qu'une scène. Elle est aussi actante, créant elle-même l'intrigue. Sorte de micro-société, elle délimite ses propres frontières entre espace privé et espace public, possédant ses propres forces de l'ordre, sa poste, ses cheminots (Ribeill, 1984), se déployant dans un système spatial total, du réseau au compartiment. Monde en soi, la gare est humaine et technique, automatisée et sociale, normée et marginale en même temps. Certes l'acception d'une société plurielle y est quelque peu bousculée. En France, le sol est propriété de I'État, la gare est concédée à une unique compagnie ferroviaire elle-même constituée en Société Anonyme. Ce lieu confine à l'empire, ce que traduisent d'ailleurs certaines pratiques discutables. Les premières gares enferment leurs voyageurs, la défiance vis-à-vis du public y est légion et la surveillance est active, les catégorisations sont toujours à l'œuvre. En même temps, comme dans toute société, s'y créent des marges actives qui la rendent plus complexe.

Mi-urbaine, mi-industrielle, la gare figure un ailleurs qui vient troubler l'expérience de la ville. Le thème de la déshumanisation par l'ébranlement des sens, l'arrachement-déracinement, est omniprésent dans la littérature consacrée aux gares. "Le train démarre lentement et toutes vitres baissées, nous pouvons voir disparaître l'être concerné : un arrachement d'autant plus douloureux qu'il se produit peu à peu au fil des secondes » (Sansot, 1971, p. 85). Constituée des arrière-cours, des voies et des hangars, des tuyauteries d'alimentation, la gare serait une machinerie qui broierait l'urbain. Coupable de " ferroviariser » (8) la ville, elle est, dès la Monarchie de Juillet (Bowie, 2003), stigmatisée comme l'emblème de l'industrialisation, voire de l'américanisation de la société (9). Pour civiliser la gare, compagnies, architectes et ingénieurs vont la figurer comme ville. Sa monumentalité s'affirme : par son faste architectural, sa modernité technique, son excitation urbaine. En même temps, cet effort d'assimilation urbaine contribue paradoxalement à affirmer la splendide autosuffisance de la gare. La ville marque la gare de son empreinte tandis que la gare s'affranchit de la ville. Comme l'exprimait Sansot (1971) : " Les lieux privilégiés représentent, d'une façon éminente, la cité, mais parce qu'ils possèdent une forte personnalité, ils lui font du même coup contrepoids, ils nous en absentent par quelques côtés. La gare nous ouvre à la ville et, en même temps, elle constitue un univers qui se suffit à lui-même, dans lequel on peut entrer pour échapper à une ville que l'on ressent comme intolérable. » (p. 90).

Une dernière frontière se dessine enfin dans l'hybridation qui s'opère entre l'uniformisation et la différenciation des gares. Celles-ci semblent posséder un air de famille, que confirment et renforcent les nombreux ouvrages typologiques consacrés à I'architecture des gares. Il est vrai que si l'on observe notre environnement contemporain, les gares présentent des modalités d'aménagement communes, des caractéristiques de gestion assez semblables ; elles semblent utilisées d'une manière de plus en plus standardisée par les usagers, les couches sémiotiques du langage que constituent la signalisation ou les franchises commerciales ne faisant qu'ajouter à cette internationalisation des grandes gares capitales. Les modèles convergent parce 
qu'ils circulent, se diffusent et ce processus peut lui aussi être inscrit dans une historicité. En même temps, un deuxième mouvement contraire semble être à l'œuvre, celui de l'individualisation des projets et des objets, qui procède du fait que la gare est avant tout inscrite dans un espace concret et contextualisé. Le territoire imprime sa marque sur l'équipement, au travers des forces politiques, économiques, culturelles et sociales qui infléchissent sa configuration et ses usages. Autrement dit, la convergence des modèles s'accompagne d'une différenciation des ancrages.

\section{LA TRANSDISCIPLINARITÉ DU TERRAIN FERROVIAIRE}

Les contributeurs au séminaire et à ce numéro ont été sollicités sur plusieurs critères. D'abord, sur l'apport de leur discipline et de leur problématique de recherche à la compréhension du couple gare-urbain : historique sur la construction d'une urbanité, politiste sur les controverses quant au devenir patrimonial des gares, socio-géographique sur les pratiques spatiales, sociologique sur la question de la surveillance. À chaque fois, ces regards observent le couple à partir d'une perspective, l'analysant depuis leur discipline et composant par là même diverses faces du kaléidoscope qu'il forme. Le terrain ferroviaire n'est pas un seul terrain, mais une géographie protéiforme dont le relief se modifie selon l'horizon scruté et la problématique explorée. En retour, et c'est aussi à cette question que s'adressent les articles, la dimension transdisciplinaire et transcalaire de l'objet interroge la recherche urbaine, ses questionnements, ses méthodes, ses corpus, ses terrains, ses finalités.

Ce numéro s'ouvre sur l'article de Stéphanie Sauget qui circonscrit la gare comme horizon d'attente, comme espace de pratiques. Partant de la situation parisienne au XIX ${ }^{\mathrm{e}}$ siècle, elle investit la notion d'urbanité dont les expériences sont renouvelées par le "choc » mécanique, dimensionnel et de masse que provoque l'arrivée du chemin de fer. Faisant l'hypothèse $d^{\prime}$ 'un processus dual de civilisation de l'espace ferroviaire et de renouvellement de l'expérience urbaine - ce qu'elle nomme la ferroviarisation de la ville -, elle interroge le rôle des imaginaires sociaux dans la construction urbaine de la gare.

S'attachant à l'icône ferroviaire la plus filmée au monde, Étienne Riot revient sur le long et controversé processus de patrimonialisation de Grand Central Station à New York, déclenché au début des années 1960 par un projet de destruction partielle menaçant l'intégrité de la gare, résolu en 1978 à l'issue d'une joute finale devant la Cour Suprême des États-Unis.
L'analyse des enjeux, des échelles et des intérêts en présence permet de montrer comment une controverse locale portant sur le destin d'un patrimoine du futur se transforme en un tournant juridique historique au plan national. Elle place surtout la gare dans un registre symbolique qui l'inscrit, au prisme du débat patrimonial, dans le creuset de l'imaginaire national.

Marion Tillous propose de déplacer le regard sur l'espace du réseau souterrain du métro parisien, qu'elle considère comme territoire en soi, qui façonne des pratiques spatiales, des interactions sociales, des appropriations culturelles. S'inscrivant et se distanciant de la notion d'espace public introduite par Isaac Joseph lorsqu'il était associé au cours des années 1980 à la mission prospective de la RATP, elle s'attache à définir la nature de cette territorialité, montrant que les espaces de transport de masse, souvent vus comme anonymes et impersonnels, sont aussi des espaces d'attachement, à l'articulation entre espace public et espace familier.

S'appuyant sur l'exemple de la Gare du Nord, Florent Castagnino investit la gare comme un « laboratoire de gouvernementalité » en examinant la façon dont s'y développent les pratiques de surveillance, à l'articulation entre logiques propres à l'espace ferroviaire et objectifs de sûreté. La tension entre les deux mouvements historiques que forment la libération du mouvement de l'individu et sa mise sous surveillance trouve ici un terrain de prédilection qui révèle les termes de la différenciation ou plutôt de la catégorisation sociale des usagers.

L'apparent éclectisme des horizons et des sujets que brosse ce panel d'articles participe de l'approche heuristique choisie à l'origine du séminaire doctoral. En faisant s'entrecroiser des points de vue, des approches disciplinaires, des terrains différents, la démarche fait l'hypothèse que la coopération de mondes hétérogènes permet des éclairages nouveaux. Dans cette perspective, ce dossier s'attache à investir la gare comme macromonde dans un micromonde. Comme l'exprime le personnage du beau film de Claire Simon (2013 a), lui-même doctorant en sociologie, la gare est un «village global ». Enveloppe spatiale, architecturale, politique, symbolique, la gare condense, aspire et renouvelle l'expérience sociale de I'individu urbain. Cette unité de lieu n'en fait pas un non-lieu mais plutôt un lieu total, dont le périmètre se redéfinit sans cesse tant il aspire - sans toutefois pleinement y parvenir - à englober à chaque fois l'essence de la ville. Dès lors la relation fusionnelle que nouent la gare et la ville est toujours en mouvement, tant les deux souhaitent se posséder l'une l'autre 
comme inextricablement liées, alors qu'en même temps, elles veulent exister à part entière comme extérieures l'une à l'autre.

Nacima Baron est professeure de Géographie à l'Université Paris-Est Marne la Vallée, directrice adjointe du Master en Urbanisme et Aménagement de l'École d'Urbanisme de Paris, directrice de la Chaire Gare et membre du laboratoire Ville Mobilité Transport (UMR-T 9403, UPEM, École des Ponts, IFSTTAR, UPE). Ses recherches portent sur les politiques territoriales d'aménagement, de mobilité et de tourisme en France et dans les métropoles espagnoles. nacimabaron@gmail.com
S'attacher à cette frontière mouvante et perméable permet d'en mesurer les horizons potentiels.

Nathalie Roseau, polytechnicienne, architecte et docteur en urbanisme, est chercheure au Laboratoire Techniques, Territoires et Société (UMR 8134, École des Ponts, UPEM, CNRS, UPE), maître de conférences à l'École nationale des ponts et chaussées et directrice du Mastère Aménagement et maîtrise d'ouvrage urbaine. Ses recherches portent sur les fonctions de l'imaginaire dans la fabrique de l'espace urbain ainsi que sur les représentations et pratiques de la ville des flux. nathalie.roseau@enpc.fr

\section{Notes}

(1) Voir par exemple Meunier M., 29 octobre 2014, "Combat environnemental : à Stuttgart, la gare de la discorde", La Croix.

(2) La chaire "Réinventer les gares au XXle siècle » a été créée en juin 2012 au sein de l'École nationale des ponts et chaussées. Si la chaire émane d'une offre de partenariat de la cinquième branche de la SNCF, Gares \& Connexions, alors en pleine phase de structuration, il est vite apparu nécessaire de dépasser le champ de l'expertise pour organiser un lieu d'échange et de construction collective à disposition des jeunes chercheurs sur le moyen terme. Ce lieu a donc pris la forme d'un séminaire associant les forces de deux laboratoires (LATTS et LVMT) et largement ouvert à d'autres partenaires, en particulier l'École Doctorale Ville Transport Territoire de I'Université Paris-Est, pour construire un rendez-vous régulier.

(3) Le séminaire a invité, autour de huit thèmes, les chercheurs suivants que nous remercions pour leur contribution : «Espaces publics sous tension » avec Florent Castagnino et Armando Ortuno Padilla, 23 avril 2013 ; «Individus et expériences de l'urbanité » avec Stéphanie Sauget et Suzanne Van Weddingen, 22 juin 2013 ; "Ancrages et circulations » avec Éloïse Libourel, Étienne Riot et Richard Zelezny, 10 septembre 2013 ; "Nouvelles techniques, nouvelles pratiques?» avec Simone Morgagni et Marion Tillous, 25 novembre 2013; "Images, imaginaires et stratégies urbaines » avec Raphaële
Bidault-Waddington, Karen Bowie et Cristiana Mazzoni, 11 mars 2014 ; "Échelles des gares » avec Félix Adisson et Juliette Maulat, 11 juin 2014 ; «La métropole en périphérie, Renouvellement des gares périurbaines en Île-de-France » avec Élise Avide et Julie Perrin, 24 mars 2015 ; « Une fabrique citoyenne des gares ? Contestation, rébellion et retournements de valeurs » avec Aurélie Delage et Anaïs Volin, 9 juin 2015.

(4) Le trafic de la gare de Juvisy-sur-Orge est plus important que celui de la gare de Rennes.

(5) Lire la revue en ligne Des rails, la revue de I'Imaginaire ferroviaire, disponible à l'adresse : http://desrails.free.fret (consulté le 4 novembre 2015).

(6) Émile Zola était un grand ami du peintre Auguste Renoir, père de Jean Renoir. Lire de Lucie Wibault, "La bête humaine de Jean Renoir ", disponible en ligne à l'adresse suivante: http://www.iletaitunefoislecinema.com/chronique/1942/ la-bete-humaine-de-jean-renoir (consulté le 4 novembre 2015)

(7) "Scènes de gare ", Le Monde, 27 avril 2013, p. 8. Article publié en I'honneur du centenaire de la création de la gare actuelle de Grand Central Station et qui traitait de son rapport au cinéma.

(8) Nous empruntons ce néologisme à : Sauget, 2009

(9) On pense au rôle des gares pour les expositions universelles, mais aussi au jeu d'émulation entre les villes européennes. À partir de 1851, Paris est annoncée comme la Ville Lumière.

\section{BibliograPHIE}

Annales des Ponts et Chaussées, avril 1999, Dossier « Gares et urbanisation », n 89 .

ANNALES DE LA RECHERCHE URBAINE, 1996, Dossier « Gares en mouvement » Joseph I. (dir.), $\mathrm{n}^{\circ} 71$.

Aveline-Dubach N., 2003, La ville et le rail au Japon, l'expansion des groupes ferroviaires privés à Tokyo et Osaka, Paris : CNRS Éditions.
Belle J., Leighton M.R., 2012, Grand Central, Gateway to a million lives, New York : Norton and company.

BowIE K. (dir.), 1987, Les grandes gares parisiennes du XIXe siècle, Paris : Délégation à I'Action artistique de la Ville de Paris.

BOWIE K., 1996. De la gare du XIXe siècle au lieu-mouvement. Évolution ou rupture?, Les Annales de la recherche urbaine, $\mathrm{n}^{\circ} 71$, p. 14-23. 
BowiE K., 2003, Débats sous la monarchie de Juillet, in : Bowie K., Texier S. (dir.), Paris et ses chemins de fer, Paris : Action artistique de la ville de Paris, p. 36-51.

Bowie K., TeXier S. (dir.), 2003, Paris et ses chemins de fer, Paris : Action artistique de la Ville de Paris.

Bradley S., 2011, St Pancras station, Londres : Profile Books.

Castagnino F., 2016, Séparer pour mieux surveiller. Spatialité des risques et pratiques de surveillance en Gare du Nord, Flux 2016/1-2 (n 103-104), p. 48-60.

Chéroux C., 1996, Vues du train, Vision et mobilité au XIX ${ }^{\mathrm{e}}$ siècle, Etudes photographiques, $\mathrm{n}^{\circ} 1$, [en ligne] (consulté le 9 mars 2016). Disponible à l'adresse: https://etudesphotographiques.revues.org/101

Clozier R., 1940, La Gare du Nord, Paris : Baillière et Fils.

Dethier J., 1978, Le temps des gares, Paris : Centre de création industrielle.

Dethier J., 1981, All stations, a Journey through 150 years of Railway history, Londres: The Science Museum.

Desportes M., 2005, Paysages en mouvement, Perception de I'espace et transports, XVIII'-XXe siècles, Paris : Gallimard.

Doumas E., 2008, Diversification des activités et privatisation des gares japonaises, Thèse de doctorat en économie, sous la dir. d'André de Palma, Université Paris Est - École nationale des ponts et chaussées.

Dupur G., 1993, Les stations nodales du métro de Paris : le réseau métropolitain et la revanche de l'histoire, Annales de géographie, $\mathrm{n}^{\circ} 102$, p. 17-31.

Duthilleul J.-M., Tricaud E., 1999, Parcours 1988-1998, Paris : Agence des gares et AREP.

Guide Gallimard, 2000, La France des Gares, Paris : Gallimard.

Grillet-Aubert A. (dir.), 2015, La desserte ferroviaire des territoires périurbains, Construire autour des gares, Bruxelles/ Milan/Paris/Washington, Paris: Éditions Recherches, ENSAPB.

JOSEPH I. (dir.), 1995, Gare du Nord mode d'emploi, Paris : Éditions Recherches, en coédition avec le Plan Urbain, la RATP et la SNCF.

JosepH I., 1996, Ariane ou I'opportunisme méthodique, Les Annales de la recherche urbaine, $\mathrm{n}^{\circ} 71$, p. 5-13.

JOSEPH I. (dir.), 1999, Villes en gares, La Tour-d'Aigues : Éditions de l'Aube.

Lemolne B., 1994, La gare, temple de l'industrialisation et porte de voyage, Architecture Intérieure Créé, n²62, p. 24-37.

Mazzoni C., 2001, Gares, architectures 1990-2010, Arles : Actes Sud.

OfFNer J.-M., 1993, Les « effets structurants » du transport: mythe politique, mystification scientifique, Espace géographique, vol. 22, $\mathrm{n}^{\circ} 3$, p. 233-242.
Puccı P., 1999, Interconnexion et rôle des nœuds d'infrastructures, du sectoriel au général, Flux, vol. 15, n 38, p. 30-38.

Ribeill G., 1984, Les cheminots, Paris : La Découverte.

RibeiLl G., 1993, La révolution ferroviaire, la formation des compagnies de chemin de fer (1823-1870), Paris : Belin.

Ribeill G., 1996, Les métamorphoses de la grande gare française, Les Annales de la recherche urbaine, $\mathrm{n}^{\circ} 71$, p. 55-65.

Rıot É., 2015, L'agencement des grandes gares historiques pour le marché ferroviaire européen, L'intégration des principes concurrentiels dans l'aménagement et la gestion des gares de London St Pancras, Paris Nord et Milano Centrale, Thèse de doctorat en aménagement, sous la direction de Nacima Baron et Vincent Kauffmann, Université Paris Est.

Rıот É., 2016, Un tournant patrimonial : New York Grand Central (1903-1978), Flux 2016/1-2 (n 103-104), p. 25-35.

Roseau N., 2012, Aerocity, Quand I'avion fait la ville, Marseille : Parenthèses.

SANDER A., 1995, Les points de réseaux comme formes urbaines, Morphogenèse et enjeux de conception, Thèse de doctorat en aménagement sous la direction de Gabriel Dupuy, Université Paris XII - Val de Marne.

Sansot P., 1971, Poétique de la ville, Paris : Klincksieck.

SAuGET S., 2009, À la recherche des pas perdus, Dans la matrice des gares parisiennes, 1837-1914, Paris : Tallandier.

Sauget S., 2016, Les gares parisiennes dans les expériences d'urbanité (1837-1914), Flux 2016/1-2 (n 103-104), p. 13-24.

SchivelbusCh W., 1990, Histoire des voyages en train, Paris: Gallimard, Collection Le Promeneur.

Simon C., 2013, Gare du Nord, Film, Production : Les films d'ici et productions Thalie, Durée : 1h59min

Simon C., 2013, Géographie humaine, Documentaire, Production : les films d'ici (Richard Copans), Durée : 1h40 min

TERrin J.-J. (sous la direction de), 2011, Gares et dynamiques urbaines, Les enjeux de la grande vitesse, Marseille : Parenthèses.

Tillous M., 2016, Le métro comme territoire : à I'articulation entre I'espace public et l'espace familier, Flux 2016/1-2 ( $\mathrm{n}^{\circ}$ 103-104), p. 36-47.

TRAVERSES, 1978, Dossier "Réseaux, modèles ferroviaires", $\mathrm{n}^{\circ}$ 13, Paris : Centre de Création Industrielle.

VAN WedDINGEN S., 2004, La rêverie ferroviaire dans la littérature fantastique européenne, Thèse de doctorat en littérature française et comparée, sous la direction de Erik Lysoe, Université de Haute-Alsace.

WuCHERPFENNIG C., 2006, Bahnhof: (Stadt)gesellschaftlicher Mikrokosmos im Wandel. Eine "neue kulturgeographische" Analyse, vol. 22 de Wahrnehmungsgeographische Studien, Oldenburg : BIS-Verlag der Univ. Oldenburg. 